\title{
At the heart of innovation: cardiac imaging in 2019
}

\author{
Martin J. Willemink ${ }^{1}$ (D)
}

Received: 17 April 2020 / Revised: 6 June 2020 / Accepted: 23 July 2020 / Published online: 1 August 2020

(C) European Society of Radiology 2020

Cardiac imaging is a rapidly evolving space with numerous substantial developments. New imaging technologies were developed over the last decades, allowing for improved morphologic and functional assessment of the heart. The year 2019 was a particularly exciting year for cardiac imaging.

The role of coronary CTA became more prominent due to the 2019 European Society of Cardiology guidelines for the diagnosis and management of chronic coronary syndromes [1]. Non-invasive functional imaging for myocardial ischemia or coronary CTA is now recommended as the initial test for diagnosis of coronary artery disease in symptomatic patients if obstructive coronary artery disease could not be excluded by clinical assessment alone. Although coronary CTA is known for its high sensitivity, the specificity is less optimal. Functional techniques such as CT-based fractional flow reserve [2-6] and myocardial CT perfusion [7-9] have the potential to improve detection of functionally significant coronary artery disease [10]. In addition, van Hamersvelt and colleagues [11] showed that the diagnostic accuracy for identification of functionally significant stenosis in patients with intermediate-degree coronary stenosis on CTA improved by a deep learning approach of the left ventricular myocardium. The deep learning method first automatically segmented the left ventricular myocardium, subsequently characterized it, and finally classified patients into those with or without functionally significant coronary artery stenosis.

Other journals also published important cardiovascular imaging studies in 2019. Mack et al [12] compared transcatheter aortic valve replacement (TAVR) and surgical valve replacement in low-risk patients with severe aortic stenosis (PARTNER 3 trial). Their New England Journal of Medicine paper showed a lower rate of a composite of death, stroke, and rehospitalization 1 year after TAVR. With potentially expanding TAVR indications to lower risk patients, adequate pre-TAVR imaging analysis becomes even more

Martin J. Willemink

m.j.willemink@stanford.edu

1 Department of Radiology, Stanford University School of Medicine, 300 Pasteur Drive, S-072, Stanford, CA 94305-5105, USA important. Francone et al [13] published a European Society of Cardiovascular Radiology (ESCR) consensus document for $\mathrm{CT}$ and MR imaging prior to TAVR. The authors provide a reference document for $\mathrm{CT}$ and MR acquisition techniques, which takes into account the significant technological variation of scanners. They also reviewed required measurements and defined a step-by-step approach for these measurements. Lastly, they proposed a CT/MR reporting template to stimulate consistent communication between different hospitals and between medical specialists involved in the procedural planning of TAVRs.

One of the 10 most downloaded cardiovascular European Radiology publications from 2019 was about diagnosing infective endocarditis. Echocardiography is currently the reference standard. Hryniewiecki et al [14] evaluated the usefulness of cardiac CT in the diagnosis of infective endocarditis and its perivalvular complications and found that $\mathrm{CT}$ is a useful modality in addition to echocardiography. The overall sensitivity of combined echocardiography and cardiac CT improved especially for detection of abscesses and pseudo-aneurysms.

Another important study in the cardiac imaging field came from the multicenter cardiac MRCT Registry of the European Society of Cardiovascular Radiology (ESCR). In general, gadolinium-based contrast agents for MR imaging are considered relatively safe. When administered for cardiac MR, gadolinium is routinely combined with pharmacological stressors such as regadenoson and adenosine, which may influence the incidence of acute adverse events. Uhlig and colleagues [15] evaluated the likelihood of gadolinium-associated acute adverse events in cardiac MR in a large registry of 72,839 patients. The large sample size allowed for statistical adjustment for potential confounders such as stress test imaging, main indications, age, and sex. The results indicated that acute adverse event rates in cardiac MR with gadolinium-based contrast were low $(0.36 \%)$, especially for severe adverse events $(0.033 \%)$. Stress cardiac MR resulted in more frequent mild and moderate adverse events.

The recently published results of the Dutch-Belgian lung cancer screening trial (Nelson trial) indicated that lung cancer mortality was reduced in the low-dose CT screening arm [16]. Since the National Lung Screening Trial (NLST) in the USA 
showed similar results in 2011 [17], one can expect that the number of CT scans of the chest will increase. Even without the potential launch of new lung cancer screening CT programs, the annual number of CT scans is increasing yearly with $6.5 \%$ [18]. The probability that chest CT scans will reveal cardiovascular findings is substantial, especially in a high-risk lung cancer screening population. Krueger and colleagues [19] realized the importance of incidental cardiac disease on un-gated chest CT scans and published a pictorial review for non-cardiovascular radiologists in Insights into Imaging on this topic. Among others, they describe cardiac shunts, chamber enlargements, and diseases of the valves, coronary arteries, cardiac walls, myocardium, and pericardium. The review paper does not only describe these clinically significant cardiac findings as seen on chest $\mathrm{CT}$, but also explains further management strategies.

These papers are just a small sample of the incredible number of innovative studies on cardiac imaging that was published in our journal and other journals in the year 2019. We are excited to see this trend continuing in 2020 with more important cardiac imaging studies focusing on novel developments ranging from improved functional coronary assessment to artificial intelligence.

Funding information The author states that this work has not received any funding.

\section{Compliance with ethical standards}

Guarantor The scientific guarantor of this publication is Martin J. Willemink.

Conflict of interest Activities related to the present article: none. Activities not related to the present article: research grants from American Heart Association, Philips Healthcare, and Stanford University, and consulting for Arterys, Inc. Co-founder/shareholder of Segmed, Inc. Other relationships: disclosed no relevant relationships.

Statistics and biometry No complex statistical methods were necessary for this paper.

Informed consent Written informed consent was not required for this study because it is an Editorial.

Ethical approval Institutional Review Board approval was not required because it is an Editorial.

\section{Methodology}

- Editorial

\section{References}

1. Knuuti J, Wijns W, Saraste A et al (2020) 2019 ESC guidelines for the diagnosis and management of chronic coronary syndromes. Eur Heart J 41(3):407-477

2. von Knebel Doeberitz PL, De Cecco CN, Schoepf UJ et al (2019) Coronary CT angiography-derived plaque quantification with artificial intelligence CT fractional flow reserve for the identification of lesion-specific ischemia. Eur Radiol 29(5): 2378-2387

3. Yu M, Lu Z, Shen $\mathrm{C}$ et al (2019) The best predictor of ischemic coronary stenosis: subtended myocardial volume, machine learning-based FFRCT, or high-risk plaque features? Eur Radiol 29(7):3647-3657

4. Liu X, Wang Y, Zhang H et al (2019) Evaluation of fractional flow reserve in patients with stable angina: can CT compete with angiography? Eur Radiol 29(7):3669-3677

5. Zhuang B, Wang S, Zhao S, Lu M (2020) Computed tomography angiography-derived fractional flow reserve (CT-FFR) for the detection of myocardial ischemia with invasive fractional flow reserve as reference: systematic review and meta-analysis. Eur Radiol 30(2):712-725

6. Zhou F, Tang CX, Schoepf UJ et al (2019) Fractional flow reserve derived from CCTA may have a prognostic role in myocardial bridging. Eur Radiol 29(6):3017-3026

7. Hubbard L, Malkasian S, Zhao Y, Abbona P, Molloi S (2019) Timing optimization of low-dose first-pass analysis dynamic CT myocardial perfusion measurement: validation in a swine model. Eur Radiol Exp 3(1):16

8. Lukas S, Feger S, Rief M, Zimmermann E, Dewey M (2019) Noise reduction and motion elimination in low-dose 4D myocardial computed tomography perfusion (CTP): preliminary clinical evaluation of the ASTRA4D algorithm. Eur Radiol 29(9):4572-4582

9. Dai X, Yu M, Pan J et al (2019) Image quality and diagnostic accuracy of coronary CT angiography derived from low-dose dynamic CT myocardial perfusion: a feasibility study with comparison to invasive coronary angiography. Eur Radiol 29(8):4349 4356

10. Hamon M, Geindreau D, Guittet L, Bauters C, Hamon M (2019) Additional diagnostic value of new $\mathrm{CT}$ imaging techniques for the functional assessment of coronary artery disease: a meta-analysis. Eur Radiol 29(6):3044-3061

11. van Hamersvelt RW, Zreik M, Voskuil M, Viergever MA, Isgum I, Leiner T (2019) Deep learning analysis of left ventricular myocardium in $\mathrm{CT}$ angiographic intermediate-degree coronary stenosis improves the diagnostic accuracy for identification of functionally significant stenosis. Eur Radiol 29(5):23502359

12. Mack MJ, Leon MB, Thourani VH et al (2019) Transcatheter aortic-valve replacement with a balloon-expandable valve in lowrisk patients. N Engl J Med 380(18):1695-1705

13. Francone M, Budde RPJ, Bremerich J et al (2019) CT and MR imaging prior to transcatheter aortic valve implantation: standardisation of scanning protocols, measurements and reporting-a consensus document by the European Society of Cardiovascular Radiology (ESCR). Eur Radiol 30(5):26272650

14. Hryniewiecki T, Zatorska K, Abramczuk E et al (2019) The usefulness of cardiac CT in the diagnosis of perivalvular complications in patients with infective endocarditis. Eur Radiol 29(8):4368-4376

15. Uhlig J, Lucke C, Vliegenthart R et al (2019) Acute adverse events in cardiac MR imaging with gadolinium-based contrast agents: results from the European Society of Cardiovascular Radiology (ESCR) MRCT registry in 72,839 patients. Eur Radiol 29(7): 3686-3695

16. de Koning HJ, van der Aalst CM, de Jong PA et al (2020) Reduced lung-cancer mortality with volume $\mathrm{CT}$ screening in a randomized trial. N Engl J Med 382(6):503-513

17. National Lung Screening Trial Research T, Aberle DR, Adams AM et al (2011) Reduced lung-cancer mortality with low-dose 
computed tomographic screening. N Engl J Med 365(5):395409

18. Willemink MJ, Noel PB (2019) The evolution of image reconstruction for CT-from filtered back projection to artificial intelligence. Eur Radiol 29(5):2185-2195
19. Krueger M, Cronin P, Sayyouh M, Kelly AM (2019) Significant incidental cardiac disease on thoracic CT: what the general radiologist needs to know. Insights Imaging 10(1):10

Publisher's note Springer Nature remains neutral with regard to jurisdictional claims in published maps and institutional affiliations. 